\title{
Dynamics of a two-neuron system with discrete and distributed delays
}

\author{
Shigui Ruan ${ }^{\mathrm{a}, *, 1}$, Rami S. Filfil ${ }^{\mathrm{b}}$ \\ a Department of Mathematics, University of Miami, Carol Gables, FL 33124-4250, USA \\ ${ }^{\mathrm{b}}$ Department of Mathematics and Statistics, Dalhousie University, Halifax, NS, Canada B3H $3 J 5$
}

Received 28 October 2002; received in revised form 6 November 2003; accepted 1 December 2003

Communicated by A. Doelman

\begin{abstract}
In this paper we consider a two-neuron network model with multiple discrete and distributed delays, where the distributed delays describe the neural feedback and the discrete delays describe the neural interaction history. Three special cases of the artificial neural network model are considered. The first case corresponds to two neural interactions with instantaneous feedback for each neuron and neural interaction history. The second case corresponds to two neural interactions with delayed neural feedback and no neural interaction history. The last case corresponds to two neural interactions with delayed neural feedback and neural interaction history. Local stability analyses are carried out for all three cases. Numerical simulations are performed to illustrate the obtained results.
\end{abstract}

(C) 2003 Elsevier B.V. All rights reserved.

Keywords: Two-neuron system; Discrete and distributed delays; Neural feedback; Neural interaction history

\section{Introduction}

Neural networks are complex and large-scale nonlinear dynamical systems, while the dynamics of the delayed neural network are even richer and more complicated [28]. For simplicity, some researchers have suggested studying the dynamical behavior of simple systems. This is very useful since the complexity found may be carried over to large networks. Babcock and Westervelt [1] studied a two-neuron network model with two delays and showed that the model exhibits interesting dynamics including underdamped ringing transients, stable and unstable limit cycles, etc. When the two delays are equal, Gopalsamy and Leung [11] showed that under certain conditions the delay induces Hopf bifurcation. Olien and Belair [21] investigated the stability of a two-neuron system with discrete time delays and no self-connections. Wei and Ruan [27] analyzed a simple neural network with two delays. For the case with self-connection, they showed that Hopf bifurcation occurs when the sum of the two delays passes through

\footnotetext{
* Corresponding author. Tel.: +1-305-284-2312; fax: +1-305-284-2848.

E-mail address: ruan@math.miami.edu (S. Ruan).

${ }^{1}$ On leave from the Department of Mathematics and Statistics, Dalhousie University, Halifax, NS, Canada.
} 
a sequence of critical values. For other related study on two-neuron networks with discrete delays, we refer to $[3,7,9,23]$ and the references cited therein.

The use of constant discrete delays in modeling delayed neural feedback provides a good approximation to simple circuits consisting of a small number of neurons. However, neural networks usually have spatial extent due to the presence of a multitude of parallel pathways with a variety of axon sizes and lengths. Thus, there will be a distribution of propagation delays. This means that the signal propagation is no longer instantaneous and is better represented by a model with distributed delays. Tank and Hopfield [25] have proposed a neural circuit with distributed delays. Gopalsamy and He [10] investigated the stability of neural networks with distributed delays. Liao et al. [15] studied the stability switches and bifurcation of a two-neuron system with a distributed time delay. They showed the existence of Hopf bifurcation using the mean delay as the bifurcation parameter. See also $[8,16]$ and the references therein.

Cowan [5] suggested that the neuron firing rate depends not only on the external input but also on the neural interaction within the network. Sokolove [24] modified the neural model by considering neural adaptation. Ogûztöreli [19] modified the neural model to include neural interaction history and adapted the model to describe a network of a finite number of neurons. This latter formulation assumes that the cell's response is driven by the input signal, the cell's history, and the cell's coupling to other cells undergoing similar or different stimulation. Taking account of these three factors, the neural network model will have both discrete and distributed delays.

In this paper we consider a two-neuron network model with multiple discrete and distributed delays, where the distributed delays describe the neural feedback and the discrete delays describe the neural interaction history. Three special cases of the neural network model are analyzed in Sections 3-5. The first case corresponds to two neural interactions with instantaneous feedback for each neuron and neural interaction history. The second case corresponds to two neural interactions with delayed neural feedback and no neural interaction history. The last case corresponds to two neural interactions with delayed neural feedback and neural interaction history. Local stability and bifurcation analyses are performed for all three cases. To verify the theoretical analysis, numerical simulations are given in Section 6. Finally, a conclusion is drawn in Section 7.

\section{The general model}

The general neural network model with delayed neural feedback and neural interaction history is described by a system of nonlinear integro-differential difference equations of the form

$$
\begin{aligned}
& \frac{1}{a_{i 0}} \frac{\mathrm{d} x_{i}(t)}{\mathrm{d} t}+x_{i}(t)=F\left\{f_{i}(t)+\sum_{j=1}^{n} c_{i j} x_{j}\left(t-\sigma_{i j}\right)+\sum_{k=1}^{m} b_{i k} \int_{-\infty}^{t} x_{i}(\tau) K_{i k}(t-\tau) \mathrm{d} \tau\right\} \\
& \text { for } t \geq 0 \text {, where } i=1,2, \ldots, n \text {, }
\end{aligned}
$$

where $x_{i}(t)$ is the normalized firing rate of the $i$ th neuron at time $t ; n$ the number of the neurons in the network; $m$ the maximal order of the network; $a_{i 0}$ the rate constant characterized by the fact that a step change in input to the $i$ th neuron produces an exponential approach from the initial value to a steady-state firing rate with the rate constant $a_{i 0} ; b_{i k}$ an adaptation or self-inhibition factor for the $i$ th neuron in the case $b_{i k}<0$ and a self-excitation factor in the case $b_{i k}>0$ with the rate constant $a_{i 0}>0 ; c_{i j}$ the interaction coefficient denoting the influence of the $j$ th neuron to the $i$ th neuron and represents an inhibition in the case $c_{i j}<0$ and an excitation in the case $c_{i j}>0$; if the $j$ th neuron is not connected directly to the $i$ th neuron we have $c_{i j}=0$; furthermore, we always assume that $c_{i i}=0$ (for $i=1,2, \ldots, n$ ) since the self-inhibition and self-excitation in the $i$ th neuron are already considered in 
the parameters $b_{i k}$ and $a_{i 0} ; \sigma_{i j}(\geq 0)$ is the time lag occurring in the transfer of the activity of the $j$ th neuron to $i$ th neuron; we assume that $\sigma_{i i}=0$ (for $\left.i=1,2, \ldots, n\right) ; K_{i k}(t)$ the delayed feedback kernel satisfying

$$
\int_{0}^{\infty} K_{i k}(s) \mathrm{d} s=\text { const. }, \quad \int_{0}^{\infty} s K_{i k}(s) \mathrm{d} s<\infty
$$

$f_{i}(t)$ the external input to the $i$ th neuron at time $t ; F$ the neuron activation function given by

$$
F\{u\}=\frac{1}{1+\mathrm{e}^{-u}} .
$$

Let $\mathrm{BC}_{+}^{n}$ denote the Banach space of bounded continuous functions mapping from $(-\infty, 0]$ to $R_{+}^{n}$ (see [12]). By the general theory of integral differential equations (see [2,18]), for any initial data $\phi=\left(\phi_{1}, \phi_{2}, \ldots, \phi_{n}\right) \in \mathrm{BC}_{+}^{n}$, there exists a unique solution $x(\phi, t)=\left(x_{1}(\phi, t), x_{2}(\phi, t), \ldots, x_{n}(\phi, t)\right)$ for all $t \geq 0$ and $x(\phi, s)=\phi(s)$ for $s \in(-\infty, 0]$.

Model (2.1) can be easily adapted to any neural network whenever its circuits and interactions are known. This has led to the adoption of this model to represent a large number of studies $[14,19,20]$.

In the next three sections we shall consider several special cases of the neural model (2.1) with two neurons $(n=2)$ and a single feedback for each neuron $(m=1)$. Note that the simplest case in which the time lags arise in a neural way is when $n=2$ since $\sigma_{i i}=0$. Therefore, system (2.1) can be written as:

$$
\begin{aligned}
& \frac{1}{a_{10}} \frac{\mathrm{d} x_{1}(t)}{\mathrm{d} t}+x_{1}(t)=F\left\{f_{1}+c_{12} x_{2}\left(t-\sigma_{12}\right)+b_{11} \int_{-\infty}^{t} x_{1}(\tau) K_{11}(t-\tau) \mathrm{d} \tau\right\}, \\
& \frac{1}{a_{20}} \frac{\mathrm{d} x_{2}(t)}{\mathrm{d} t}+x_{2}(t)=F\left\{f_{2}+c_{21} x_{1}\left(t-\sigma_{21}\right)+b_{22} \int_{-\infty}^{t} x_{2}(\tau) K_{22}(t-\tau) \mathrm{d} \tau\right\},
\end{aligned}
$$

where $F(u)$ is given by (2.2). The initial conditions are given by

$$
x_{i}(s)=\phi_{i}(s) \quad \text { for } s \in(-\infty, 0], \quad x_{i}(+0)=\phi_{i}(0) \quad(i=1,2),
$$

where the history functions $\left(\phi_{1}(s), \phi_{2}(s)\right) \in \mathrm{BC}_{+}^{2}$ with $0 \leq \phi_{i}(t) \leq 1$. The external input functions $f_{i}(t)(i=1,2)$ are piecewise continuous for $t \geq 0$. System (2.3) admits a unique continuously differentiable solution $\left\{x_{1}(t), x_{2}(t)\right\}$ for $t \geq 0$, which depends on the parameters $a_{i k}, b_{i k}, c_{i j}, \sigma_{i j}, f_{i}$ and $\phi_{i}(t)$. If $a_{i 0}>1$, then solutions of system (2.3) with the initial conditions (2.4) always satisfy

$$
0 \leq x_{i}(t) \leq 1 \text { for } t \geq 0
$$

This can be shown by considering two time instances $t_{1}$ and $t_{2}$ such that $x_{i}\left(t_{1}\right)=0$ and $x_{i}\left(t_{2}\right)=1$. Then it follows from (2.3) that

$$
\begin{aligned}
& \frac{1}{a_{i 0}} \frac{\mathrm{d} x_{i}\left(t_{1}\right)}{\mathrm{d} t}+x_{i}\left(t_{1}\right)=F\left\{f_{i}\left(t_{1}\right)+\sum c_{i j} x_{j}\left(t_{1}-\sigma_{i j}\right)+b_{i i} \int_{-\infty}^{t_{1}} x_{i}(\tau) K_{i i}(t-\tau) \mathrm{d} \tau\right\}, \\
& \frac{1}{a_{i 0}} \frac{\mathrm{d} x_{i}\left(t_{2}\right)}{\mathrm{d} t}+x_{i}\left(t_{2}\right)=F\left\{f_{i}\left(t_{2}\right)+\sum c_{i j} x_{j}\left(t_{2}-\sigma_{i j}\right)+b_{i i} \int_{-\infty}^{t_{2}} x_{i}(\tau) K_{i i}(t-\tau) \mathrm{d} \tau\right\} .
\end{aligned}
$$

Since $a_{i 0}>0$ and $0<F\{u\}<1$ for any value of $u$, we see that

$$
\frac{\mathrm{d} x_{i}\left(t_{1}\right)}{\mathrm{d} t}>0 \text { and } \frac{\mathrm{d} x_{i}\left(t_{2}\right)}{\mathrm{d} t}<0 .
$$

This implies that $x_{i}(t)$ increases just after the instance $t=t_{1}$ and decreases just after the instance $t=t_{2}$. Since $x_{i}(+0)=\phi_{i}(0)$ and by hypothesis $0 \leq \phi_{i}(t) \leq 1$, it follows that $x_{i}(t)$ is bounded and $0 \leq x_{i}(t) \leq 1$ for $t \geq 0$. This shows that the saturation phenomenon is present in the neural activity in system (2.3). 


\section{Instantaneous neural feedback and neural interaction history}

The neural model can be simplified by considering instantaneous feedback in the neurons. This can be established by choosing the kernels in (2.3) as the delta-function

$$
K_{i i}(t-\tau)=\delta(t-\tau)=\left\{\begin{array}{ll}
0 & t \neq \tau \\
1 & t=\tau
\end{array} \quad(i=1,2) .\right.
$$

The model now takes the form

$$
\begin{aligned}
& \frac{1}{a_{10}} \frac{\mathrm{d} x_{1}(t)}{\mathrm{d} t}+x_{1}(t)=F\left\{f_{1}+c_{12} x_{2}\left(t-\sigma_{12}\right)+b_{11} x_{1}(t)\right\}, \\
& \frac{1}{a_{20}} \frac{\mathrm{d} x_{2}(t)}{\mathrm{d} t}+x_{2}(t)=F\left\{f_{2}+c_{21} x_{1}\left(t-\sigma_{21}\right)+b_{22} x_{2}(t)\right\} .
\end{aligned}
$$

Choosing the initial conditions as

$$
x_{i}(\theta)=\phi_{i}(\theta) \quad(i=1,2), \theta \in\left[-\sigma_{m}, 0\right],
$$

where $\sigma_{m}=\max \left\{\sigma_{12}, \sigma_{21}\right\}, \phi_{i} \geq 0(i=1,2)$ are continuous functions on $\left[-\sigma_{m}, 0\right]$. The steady-state solutions for (3.2) are given implicitly by

$$
x_{1}^{*}=F\left\{f_{1}+c_{12} x_{2}^{*}+b_{11} x_{1}^{*}\right\}, \quad x_{2}^{*}=F\left\{f_{2}+c_{21} x_{1}^{*}+b_{22} x_{2}^{*}\right\} .
$$

In terms of the constants $f_{1}$ and $f_{2}$, we have

$$
f_{1}=-c_{12} x_{2}^{*}-b_{11} x_{1}^{*}+\ln \left[\frac{x_{1}^{*}}{1-x_{1}^{*}}\right], \quad f_{2}=-c_{21} x_{1}^{*}-b_{22} x_{2}^{*}+\ln \left[\frac{x_{2}^{*}}{1-x_{2}^{*}}\right] .
$$

Let

$$
x_{i}=x_{i}^{*}+X_{i} \quad(i=1,2) .
$$

The linearized system takes the form

$$
\begin{aligned}
& \frac{1}{a_{10}} \frac{\mathrm{d} X_{1}(t)}{\mathrm{d} t}+X_{1}(t)=q_{1}\left(c_{12} X_{2}\left(t-\sigma_{12}\right)+b_{11} X_{1}(t)\right), \\
& \frac{1}{a_{20}} \frac{\mathrm{d} X_{2}(t)}{\mathrm{d} t}+X_{2}(t)=q_{2}\left(c_{21} X_{1}\left(t-\sigma_{21}\right)+b_{22} X_{2}(t)\right),
\end{aligned}
$$

where $q_{i}=x_{i}^{*}\left(1-x_{i}^{*}\right)(i=1,2)$. The associated characteristic equation of (3.3) is

$$
\left|\begin{array}{cc}
a_{10}\left(b_{11} q_{1}-1\right)-\lambda & a_{10} q_{1} c_{12} \mathrm{e}^{-\lambda \sigma_{12}} \\
a_{20} q_{2} c_{21} \mathrm{e}^{-\lambda \sigma_{21}} & a_{20}\left(b_{22} q_{2}-1\right)-\lambda
\end{array}\right|=0 .
$$

This characteristic equation determines the local stability of the equilibrium solution. The equilibrium solution is stable if and only if all roots of (3.4) have negative real parts. For convenience, we introduce some notations:

$$
\psi_{1}=a_{10}\left(1-b_{11} q_{1}\right), \quad \psi_{2}=a_{20}\left(1-b_{22} q_{2}\right), \quad D=-a_{10} a_{20} q_{1} q_{2} c_{12} c_{21}, \quad \sigma_{s}=\sigma_{12}+\sigma_{21} .
$$

Then, Eq. (3.4) becomes

$$
\lambda^{2}+\left(\psi_{1}+\psi_{2}\right) \lambda+D \mathrm{e}^{-\lambda \sigma_{s}}+\psi_{1} \psi_{1}=0 .
$$


Eq. (3.5) has been studied in [22], see also [7,23,27]. The stability and bifurcation of system (3.1) can be summarized as the following theorem.

Theorem 3.1. For system (3.1), assume $\psi_{1}+\psi_{2}>0$ and $\psi_{1} \psi_{2}+D>0$.

(i) If $\psi_{1} \psi_{2}-D>0$, then the steady-state solution $\left(x_{1}^{*}, x_{2}^{*}\right)$ is asymptotically stable for all discrete delays $\sigma_{s}=\sigma_{12}+\sigma_{21} \geq 0$.

(ii) If $\psi_{1} \psi_{2}-D<0$, there is a critical value $\sigma_{s, 0}$ of the discrete delay so that if $\sigma_{s} \in\left[0, \sigma_{s, 0}\right)$, then the steady-state solution $\left(x_{1}^{*}, x_{2}^{*}\right)$ is asymptotically stable. If $\sigma_{s}>\sigma_{s, 0}$, then $\left(x_{1}^{*}, x_{2}^{*}\right)$ is unstable. Hopf bifurcation occurs when $\sigma_{s}=\sigma_{s, 0}$.

\section{Delayed neural feedback and no neural interaction history}

Another case of interest is when the kernels take the form of exponential function

$$
K_{i i}(t-\tau)=\mathrm{e}^{-\alpha_{i i}(t-\tau)} \quad(i=1,2),
$$

where $\alpha_{i i}>0(i=1,2)$ is the distributed delay coefficient indicating the adaptation of the neuron to its feedback. If the history of the neural interaction is not considered, the discrete delays $\sigma_{12}=\sigma_{21}=0$ in (2.3). Under these conditions, the nonlinear neural network model takes the form:

$$
\begin{aligned}
& \frac{1}{a_{10}} \frac{\mathrm{d} x_{1}(t)}{\mathrm{d} t}+x_{1}(t)=F\left\{f_{1}+c_{12} x_{2}(t)+b_{11} \int_{-\infty}^{t} x_{1}(\tau) \mathrm{e}^{-\alpha_{11}(t-\tau)} \mathrm{d} \tau\right\}, \\
& \frac{1}{a_{20}} \frac{\mathrm{d} x_{2}(t)}{\mathrm{d} t}+x_{2}(t)=F\left\{f_{2}+c_{21} x_{1}(t)+b_{22} \int_{-\infty}^{t} x_{2}(\tau) \mathrm{e}^{-\alpha_{22}(t-\tau)} \mathrm{d} \tau\right\} .
\end{aligned}
$$

Note that the phase space for system (4.1) is $\mathrm{BC}_{+}^{2}$ defined in Section 2 and the initial conditions are given in (2.4). The steady-state solution $\left(x_{1}^{*}, x_{2}^{*}\right)$ of system (4.1) with constant $f_{1}$ and $f_{2}$ is implicitly given by

$$
x_{1}^{*}=F\left\{f_{1}+c_{12} x_{2}^{*}+\frac{b_{11}}{\alpha_{11}} x_{1}^{*}\right\}, \quad x_{2}^{*}=F\left\{f_{2}+c_{21} x_{1}^{*}+\frac{b_{21}}{\alpha_{22}} x_{2}^{*}\right\} .
$$

Using the linear chain trick (see [17]) on the integro-differential equation, we define

$$
x_{i i}(t)=\int_{-\infty}^{t} x_{i}(\tau) \mathrm{e}^{-\alpha_{i i}(t-\tau)} \mathrm{d} \tau \quad(i=1,2) .
$$

The nonlinear system becomes

$$
\begin{array}{ll}
\frac{1}{a_{10}} \frac{\mathrm{d} x_{1}(t)}{\mathrm{d} t}+x_{1}(t)=F\left\{f_{1}+c_{12} x_{2}(t)+b_{11} x_{11}(t)\right\}, & \frac{\mathrm{d} x_{11}(t)}{\mathrm{d} t}=x_{1}(t)-\alpha_{11} x_{11}(t), \\
\frac{1}{a_{20}} \frac{\mathrm{d} x_{2}(t)}{\mathrm{d} t}+x_{2}(t)=F\left\{f_{2}+c_{21} x_{1}(t)+b_{22} x_{22}(t)\right\}, & \frac{\mathrm{d} x_{22}(t)}{\mathrm{d} t}=x_{2}(t)-\alpha_{22} x_{22}(t) .
\end{array}
$$

Note that the equilibrium of (4.4) takes the form ( $x_{1}^{*}, x_{11}^{*}, x_{2}^{*}, x_{22}^{*}$, where $\alpha_{11} x_{11}^{*}=x_{1}^{*}$ and $\alpha_{22} x_{22}^{*}=x_{2}^{*}$. The results in [17] indicate that the stability of $\left(x_{1}^{*}, x_{2}^{*}\right)$ of system (4.1) is equivalent to the stability of $\left(x_{1}^{*}, x_{11}^{*}, x_{2}^{*}, x_{22}^{*}\right)$ of the ODE system (4.4). To determine the stability of $\left(x_{1}^{*}, x_{11}^{*}, x_{2}^{*}, x_{22}^{*}\right)$, let

$$
x_{i}=x_{i}^{*}+X_{i} \quad(i=1,2) .
$$


The linearized system takes the form:

$$
\begin{array}{lll}
\frac{1}{a_{10}} \frac{\mathrm{d} X_{1}(t)}{\mathrm{d} t}+X_{1}(t)=q_{1}\left\{c_{12} X_{2}(t)+b_{11} X_{11}(t)\right\}, & \frac{\mathrm{d} X_{11}(t)}{\mathrm{d} t}=X_{1}(t)-\alpha_{11} X_{11}(t), \\
\frac{1}{a_{20}} \frac{\mathrm{d} X_{2}(t)}{\mathrm{d} t}+X_{2}(t)=q_{2}\left\{c_{21} X_{1}(t)+b_{22} X_{22}(t)\right\}, & \frac{\mathrm{d} X_{22}(t)}{\mathrm{d} t}=X_{2}(t)-\alpha_{22} X_{22}(t),
\end{array}
$$

where $q_{i}=x_{i}^{*}\left(1-x_{i}^{*}\right)(i=1,2)$. The associate characteristic equation of the system is

$$
\left|\begin{array}{cccc}
-a_{10}-\lambda & a_{10} b_{11} q_{1} & a_{10} c_{12} q_{1} & 1 \\
1 & -\alpha_{11}-\lambda & 0 & 0 \\
a_{20} c_{21} q_{2} & 0 & -a_{20}-\lambda & a_{20} b_{22} q_{2} \\
0 & 0 & 1 & -\alpha_{22}-\lambda
\end{array}\right|=0 .
$$

For convenience, we introduce some notations:

$$
B_{i}=a_{i 0} b_{i i} q_{i} \quad(i=1,2), \quad C=a_{10} c_{12} q_{1} a_{20} c_{21} q_{2} .
$$

Then, by expanding (4.7) and using the notation in (4.8), the characteristic equation becomes

$$
\left[\left(a_{10}+\lambda\right)\left(\alpha_{11}+\lambda\right)-B_{1}\right]\left[\left(a_{20}+\lambda\right)\left(\alpha_{22}+\lambda\right)-B_{2}\right]-C\left(\alpha_{11}+\lambda\right)\left(\alpha_{22}+\lambda\right)=0 .
$$

We now consider two special cases.

\subsection{Identical neurons}

If the two neurons in the network exhibit the same linear behavior in response to the same outputs, then we have a network of identical neurons; i.e. $a_{10}=a_{20}, \alpha_{11}=\alpha_{22}$, and $B_{1}=B_{2}$. Under these assumptions, Eq. (4.9) becomes

$$
\left[\left(a_{10}+\lambda\right)\left(\alpha_{11}+\lambda\right)-B_{1}\right]^{2}-C\left(\alpha_{11}+\lambda\right)^{2}=0,
$$

from which the four roots may be determined. If $C>0$, then Eq. (4.10) requires that either

$$
\left[\left(a_{10}+\lambda\right)\left(\alpha_{11}+\lambda\right)-B_{1}\right]=\sqrt{C}\left(\alpha_{11}+\lambda\right)
$$

or

$$
\left[\left(a_{10}+\lambda\right)\left(\alpha_{11}+\lambda\right)-B_{1}\right]=-\sqrt{C}\left(\alpha_{11}+\lambda\right) .
$$

From Eq. (4.11) the roots $\lambda_{1}$ and $\lambda_{2}$ are

$$
\lambda_{1,2}=\frac{1}{2}\left[-\left(a_{10}+\alpha_{11}-\sqrt{C}\right) \pm \sqrt{\left(a_{10}-\alpha_{11}-\sqrt{C}\right)^{2}+4 B_{1}}\right] .
$$

Note that $a_{10}, \alpha_{11}$ and $C$ are all greater than zero while $B_{1}$ can be positive or negative. Thus, if

$$
\begin{aligned}
& a_{10}+\alpha_{11}-\sqrt{C}>0, \\
& \left(a_{10}-\sqrt{C}\right) \alpha_{11}-B_{1}>0,
\end{aligned}
$$

then the real parts of $\lambda_{1}$ and $\lambda_{2}$ are negative. From Eq. (4.12) the roots $\lambda_{3}$ and $\lambda_{4}$ are

$$
\lambda_{3,4}=\frac{1}{2}\left[-\left(a_{10}+\alpha_{11}+\sqrt{C}\right) \pm \sqrt{\left(a_{10}-\alpha_{11}+\sqrt{C}\right)^{2}+4 B_{1}}\right] .
$$


Similarly, if

$$
\left(a_{10}+\sqrt{C}\right) \alpha_{11}-B_{1}>0,
$$

then the roots $\lambda_{3}$ and $\lambda_{4}$ will have real parts.

To have stability of the steady state, all three conditions, (4.14), (4.15) and (4.17), have to be satisfied. However, if any of these three conditions fails, then the steady state becomes unstable. This can occur for large positive values of $B_{1}$ (self-excitation) such that either

$$
\left(a_{10}-\sqrt{C}\right) \alpha_{11}-B_{1}<0 \text { or }\left(a_{10}+\sqrt{C}\right) \alpha_{11}-B_{1}<0 .
$$

If $B_{1}$ is negative (self-inhibition) so that (4.15) and (4.17) hold, choose the distributed delay coefficient $\alpha_{11}$ as a bifurcation parameter. If $a_{10}+\alpha_{11}-\sqrt{C}<0$ for a certain value of $\alpha_{11}$, then the steady state becomes unstable. This can be summarized into the following theorem.

Theorem 4.1. Assume the two neurons are identical. The steady state $\left(x_{1}^{*}, x_{2}^{*}\right)$ of system (4.1) is asymptotically stable if conditions (4.14), (4.15) and (4.17) are satisfied. The stability of $\left(x_{1}^{*}, x_{2}^{*}\right)$ can be lost either at the occurrence of large self-excitation in the neurons (large positive $B_{1}$ ) when (4.18) holds or in the case of self-inhibition $\left(B_{1}<0\right)$ if

$$
a_{10}+\alpha_{11}-\sqrt{C}<0
$$

for a certain value of $\alpha_{11}$.

\subsection{Non-identical neurons}

Taking the distributed delay coefficient $\alpha_{11}$ as the bifurcation parameter, we study the existence of Hopf bifurcation in system (4.1). The characteristic equation (4.9) can be written as follows:

$$
\lambda^{4}+c_{1} \lambda^{3}+c_{2} \lambda^{2}+c_{3} \lambda+c_{4}=0,
$$

where $c_{1}, c_{2}, c_{3}$, and $c_{4}$ are defined as follows:

$$
\begin{aligned}
& c_{1}\left(\alpha_{11}\right)=a_{10}+\alpha_{11}+a_{20}+\alpha_{22}>0, \\
& c_{2}\left(\alpha_{11}\right)=\left(a_{10}+\alpha_{11}\right)\left(a_{20}+\alpha_{22}\right)+a_{10} \alpha_{11}+a_{20} \alpha_{22}-B_{1}-B_{2}-C, \\
& c_{3}\left(\alpha_{11}\right)=\left(a_{10}+\alpha_{11}\right)\left(a_{20} \alpha_{22}-B_{2}\right)+\left(a_{20}+\alpha_{22}\right)\left(a_{10} \alpha_{11}-B_{1}\right)-\alpha_{11} C-\alpha_{22} C, \\
& c_{4}\left(\alpha_{11}\right)=\left(a_{10} \alpha_{11}-B_{1}\right)\left(a_{20} \alpha_{22}-B_{2}\right)-C \alpha_{11} \alpha_{22} .
\end{aligned}
$$

Assume $c_{2}\left(\alpha_{11}\right)>0, c_{3}\left(\alpha_{11}\right)>0, c_{4}\left(\alpha_{11}\right)>0$. Define

$$
\Psi\left(\alpha_{11}\right)=c_{1}\left(\alpha_{11}\right) c_{2}\left(\alpha_{11}\right) c_{3}\left(\alpha_{11}\right)-c_{3}^{2}\left(\alpha_{11}\right)-c_{1}^{2}\left(\alpha_{11}\right) c_{4}\left(\alpha_{11}\right) .
$$

The Routh-Hurwitz criterion states that the equilibrium of the system (4.6) is locally asymptotically stable if and only if $\Psi\left(\alpha_{11}\right)>0$. Note that $\Psi\left(\alpha_{11}\right)>0$ implies that $c_{1}\left(\alpha_{11}\right) c_{2}\left(\alpha_{11}\right)-c_{3}\left(\alpha_{11}\right)>0$.

Let $\lambda_{i}(i=1,2,3,4)$ be the roots of the characteristic equation (4.19). Then we have

$$
\begin{aligned}
& \lambda_{1}+\lambda_{2}+\lambda_{3}+\lambda_{4}=-c_{1}, \quad \lambda_{1} \lambda_{2}+\lambda_{1} \lambda_{3}+\lambda_{1} \lambda_{4}+\lambda_{2} \lambda_{3}+\lambda_{2} \lambda_{4}+\lambda_{3} \lambda_{4}=c_{2}, \\
& \lambda_{1} \lambda_{2} \lambda_{3}+\lambda_{1} \lambda_{3} \lambda_{4}+\lambda_{2} \lambda_{3} \lambda_{4}+\lambda_{1} \lambda_{2} \lambda_{4}=-c_{3}, \quad \lambda_{1} \lambda_{2} \lambda_{3} \lambda_{4}=c_{4} .
\end{aligned}
$$

If there exists $\alpha_{11}^{*} \in \mathbf{R}$ such that $\Psi\left(\alpha_{11}^{*}\right)=0$, then by the Routh-Hurwitz criterion at least one root, say $\lambda_{1}$, has real part equal to zero. From the fourth equation of (4.22), it follows that $\operatorname{Im} \lambda_{1}=\omega_{0} \neq 0$, and hence there is another 
root, say $\lambda_{2}$, such that $\lambda_{2}=\bar{\lambda}_{1}$. Since $\Psi\left(\alpha_{11}\right)$ is a continuous function of its roots, $\lambda_{1}$ and $\lambda_{2}$ are complex conjugates of $a_{11}$ in an open interval including $\alpha_{11}^{*}$. Therefore the equation in (4.22) have the following form at $\alpha_{11}^{*}$ :

$$
\lambda_{3}+\lambda_{4}=-c_{1}, \quad \omega_{0}^{2}+\lambda_{3} \lambda_{4}=c_{2}, \quad \omega_{0}^{2}\left(\lambda_{3}+\lambda_{4}\right)=-c_{3}, \quad \omega_{0}^{2} \lambda_{3} \lambda_{4}=c_{4} .
$$

If $\lambda_{3}$ and $\lambda_{4}$ are complex conjugate, from the first equation of (4.23), it follows that $2 \operatorname{Re} \lambda_{3}=-c_{1}<0$. If $\lambda_{3}$ and $\lambda_{4}$ are real, from the first and the fourth equations of (4.23) it follows that $\lambda_{3}<0$ and $\lambda_{4}<0$. Also, differentiating (4.11) with respect to $\alpha_{11}$, we have

$$
\frac{\mathrm{d} \lambda}{\mathrm{d} \alpha_{11}}=-\frac{\left(\mathrm{d} c_{1} / \mathrm{d} \alpha_{11}\right) \lambda^{3}+\left(\mathrm{d} c_{2} / \mathrm{d} \alpha_{11}\right) \lambda^{2}+\left(\mathrm{d} c_{3} / \mathrm{d} \alpha_{11}\right) \lambda+\mathrm{d} c_{4} / \mathrm{d} \alpha_{11}}{4 \lambda^{3}+3 c_{1} \lambda^{2}+2 c_{2} \lambda+c_{3}}
$$

Also, from Eq. (4.21), we have

$$
\frac{\mathrm{d} \Psi}{\mathrm{d} \alpha_{11}}=c_{2} c_{3} \frac{\mathrm{d} c_{1}}{\mathrm{~d} \alpha_{11}}+c_{1} c_{3} \frac{\mathrm{d} c_{2}}{\mathrm{~d} \alpha_{11}}+c_{1} c_{2} \frac{\mathrm{d} c_{3}}{\mathrm{~d} \alpha_{11}}-2 c_{3} \frac{\mathrm{d} c_{3}}{\mathrm{~d} \alpha_{11}}-2 c_{1} c_{4} \frac{\mathrm{d} c_{1}}{\mathrm{~d} \alpha_{11}}-c_{1}^{2} \frac{\mathrm{d} c_{4}}{\mathrm{~d} \alpha_{11}} .
$$

Hence

$$
\begin{aligned}
\frac{\mathrm{d}}{\mathrm{d} \alpha_{11}}[\operatorname{Re} \lambda]_{\alpha_{11}^{*}} & =\operatorname{Re}\left[-\frac{\left(\mathrm{d} c_{1} / \mathrm{d} \alpha_{11}\right) \lambda^{3}+\left(\mathrm{d} c_{2} / \mathrm{d} \alpha_{11}\right) \lambda^{2}+\left(\mathrm{d} c_{3} / \mathrm{d} \alpha_{11}\right) \lambda+\mathrm{d} c_{4} / \mathrm{d} \alpha_{11}}{4 \lambda^{3}+3 c_{1} \lambda^{2}+2 c_{2} \lambda+c_{3}}\right]_{\alpha_{11}^{*}} \\
& =\operatorname{Re}\left[-\frac{-\mathrm{i}\left(\mathrm{d} c_{1} / \mathrm{d} \alpha_{11}\right) \omega^{3}-\left(\mathrm{d} c_{2} / \mathrm{d} \alpha_{11}\right) \omega^{2}+\mathrm{i}\left(\mathrm{d} c_{3} / \mathrm{d} \alpha_{11}\right) \omega+\mathrm{d} c_{4} / \mathrm{d} \alpha_{11}}{-4 \mathrm{i} \omega^{3}-3 c_{1} \omega^{2}+2 c_{2} \mathrm{i} \omega+c_{3}}\right]_{\alpha_{11}^{*}} \\
& =-\frac{1}{2} \frac{c_{1}}{c_{1}^{3} c_{3}+\omega^{2}\left(2 c_{3}-c_{1} c_{2}\right)^{2}}\left(\frac{\mathrm{d} \Psi}{\mathrm{d} \alpha_{11}}\right)_{\alpha_{11}^{*}} .
\end{aligned}
$$

Note that (4.23) and (4.25) were used to obtain (4.26). Thus, we have the following result.

Theorem 4.2. Assume that $c_{2}\left(\alpha_{11}\right)>0, c_{3}\left(\alpha_{11}\right)>0, c_{4}\left(\alpha_{11}\right)>0$. If $\Psi\left(\alpha_{11}\right)>0$, then the steady state $\left(x_{1}^{*}, x_{2}^{*}\right)$ of system (4.1) is locally asymptotically stable. If there exists $\alpha_{11}^{*} \in \mathbf{R}$ such that $\Psi\left(\alpha_{11}^{*}\right)=0$ and $\left.\left(\mathrm{d} \Psi / \mathrm{d} \alpha_{11}\right)\right|_{\alpha_{11}^{*}} \neq 0$, then as $\alpha_{11}$ passes through $\alpha_{11}^{*}$, Hopf bifurcation occurs at the steady state $\left(x_{1}^{*}, x_{2}^{*}\right)$.

\section{Delayed neural feedback and neural interaction history}

The last case of interest is when the kernels take the form of exponential functions $K_{i i}(t-\tau)=\mathrm{e}^{-\alpha_{i i}(t-\tau)}(i=1,2)$ and when the discrete delays $\sigma_{12} \neq 0$ and $\sigma_{21} \neq 0$. This indicates the adaptation of the neuron to its feedback and the existence of neural interaction history in the model. Under these conditions, the nonlinear neural model takes the form:

$$
\begin{aligned}
& \frac{1}{a_{10}} \frac{\mathrm{d} x_{1}(t)}{\mathrm{d} t}+x_{1}(t)=F\left\{f_{1}+c_{12} x_{2}\left(t-\sigma_{12}\right)+b_{11} \int_{-\infty}^{t} x_{1}(\tau) \mathrm{e}^{-\alpha_{11}(t-\tau)} \mathrm{d} \tau\right\} \\
& \frac{1}{a_{20}} \frac{\mathrm{d} x_{2}(t)}{\mathrm{d} t}+x_{2}(t)=F\left\{f_{2}+c_{21} x_{1}\left(t-\sigma_{21}\right)+b_{22} \int_{-\infty}^{t} x_{2}(\tau) \mathrm{e}^{-\alpha_{22}(t-\tau)} \mathrm{d} \tau\right\} .
\end{aligned}
$$

The phase space for system (5.1) is $\mathrm{BC}_{+}^{2}$. The equilibrium $\left(x_{1}^{*}, x_{2}^{*}\right)$ of (4.1) is also an equilibrium of (5.1). Define

$$
x_{i i}(t)=\int_{-\infty}^{t} x_{i}(\tau) \mathrm{e}^{-\alpha_{i i}(t-\tau)} \mathrm{d} \tau \quad(i=1,2) .
$$


The nonlinear system becomes

$$
\begin{array}{lll}
\frac{1}{a_{10}} \frac{\mathrm{d} x_{1}(t)}{\mathrm{d} t}+x_{1}(t)=F\left\{f_{1}+c_{12} x_{2}\left(t-\sigma_{12}\right)+b_{11} x_{11}(t)\right\}, & \frac{\mathrm{d} x_{11}(t)}{\mathrm{d} t}=x_{1}(t)-\alpha_{11} x_{11}(t), \\
\frac{1}{a_{20}} \frac{\mathrm{d} x_{2}(t)}{\mathrm{d} t}+x_{2}(t)=F\left\{f_{2}+c_{21} x_{1}\left(t-\sigma_{21}\right)+b_{22} x_{22}(t)\right\}, & \frac{\mathrm{d} x_{22}(t)}{\mathrm{d} t}=x_{2}(t)-\alpha_{22} x_{22}(t) .
\end{array}
$$

The linearized model about the steady-state solution takes the form:

$$
\begin{array}{ll}
\frac{1}{a_{10}} \frac{\mathrm{d} X_{1}(t)}{\mathrm{d} t}+X_{1}(t)=q_{1}\left\{c_{12} X_{2}\left(t-\sigma_{12}\right)+b_{11} X_{11}(t)\right\}, & \frac{\mathrm{d} X_{11}(t)}{\mathrm{d} t}=X_{1}(t)-\alpha_{11} X_{11}(t), \\
\frac{1}{a_{20}} \frac{\mathrm{d} X_{2}(t)}{\mathrm{d} t}+X_{2}(t)=q_{2}\left\{c_{21} X_{1}\left(t-\sigma_{21}\right)+b_{22} X_{22}(t)\right\}, & \frac{\mathrm{d} X_{22}(t)}{\mathrm{d} t}=X_{2}(t)-\alpha_{22} x_{22}(t),
\end{array}
$$

where $q_{i}=x_{i}^{*}\left(1-x_{i}^{*}\right)(i=1,2)$. The associate characteristic equation of the system is

$$
\left|\begin{array}{cccc}
-a_{10}-\lambda & a_{10} b_{11} q_{1} & a_{10} c_{12} q_{1} \mathrm{e}^{-\sigma_{12} \lambda} & 0 \\
1 & -\alpha_{11}-\lambda & 0 & 0 \\
a_{20} c_{21} q_{2} \mathrm{e}^{-\sigma_{21} \lambda} & 0 & -a_{20}-\lambda & a_{20} b_{22} q_{2} \\
0 & 0 & 1 & -\alpha_{22}-\lambda
\end{array}\right|=0
$$

For convenience, we use the same notation used in Section 4

$$
B_{i}=a_{i 0} b_{i i} q_{i} \quad(i=1,2), \quad C=a_{10} c_{12} q_{1} a_{20} c_{21} q_{2} .
$$

Then the characteristic equation becomes

$$
\left[\left(a_{10}+\lambda\right)\left(\alpha_{11}+\lambda\right)-B_{1}\right]\left[\left(a_{20}+\lambda\right)\left(\alpha_{22}+\lambda\right)-B_{2}\right]-C\left(\alpha_{11}+\lambda\right)\left(\alpha_{22}+\lambda\right) \mathrm{e}^{-\left(\sigma_{12}+\sigma_{21}\right) \lambda}=0 .
$$

Assuming that the two neurons are identical, then Eq. (5.7) becomes

$$
\left[\left(a_{10}+\lambda\right)\left(\alpha_{11}+\lambda\right)-B_{1}\right]^{2}-C\left(\alpha_{11}+\lambda\right)^{2} \mathrm{e}^{-\left(\sigma_{12}+\sigma_{21}\right) \lambda}=0
$$

which can be factored into

$$
\begin{aligned}
& \left\{\left[\left(a_{10}+\lambda\right)\left(\alpha_{11}+\lambda\right)-B_{1}\right]-\sqrt{C}\left(\alpha_{11}+\lambda\right) \mathrm{e}^{-(1 / 2)\left(\sigma_{12}+\sigma_{21}\right) \lambda}\right\} \\
& \quad \times\left\{\left[\left(a_{10}+\lambda\right)\left(\alpha_{11}+\lambda\right)-B_{1}\right]+\sqrt{C}\left(\alpha_{11}+\lambda\right) \mathrm{e}^{-(1 / 2)\left(\sigma_{12}+\sigma_{21}\right) \lambda}\right\}=0 .
\end{aligned}
$$

So the characteristic equation becomes two transcendental equations

$$
\left[\left(a_{10}+\lambda\right)\left(\alpha_{11}+\lambda\right)-B_{1}\right] \pm \sqrt{C}\left(\alpha_{11}+\lambda\right) \mathrm{e}^{-(1 / 2)\left(\sigma_{12}+\sigma_{21}\right) \lambda}=0 .
$$

We shall consider the positive case first

$$
\left[\left(a_{10}+\lambda\right)\left(\alpha_{11}+\lambda\right)-B_{1}\right]+\sqrt{C}\left(\alpha_{11}+\lambda\right) \mathrm{e}^{-(1 / 2)\left(\sigma_{12}+\sigma_{21}\right) \lambda}=0 .
$$

For convenience, we introduce some notations

$$
a_{10}+\alpha_{11}=A, \quad a_{10} \alpha_{11}-B_{1}=D, \quad \sqrt{C} \alpha_{11}=E, \quad \sqrt{C}=F, \quad \frac{1}{2}\left(\sigma_{12}+\sigma_{21}\right)=\sigma .
$$




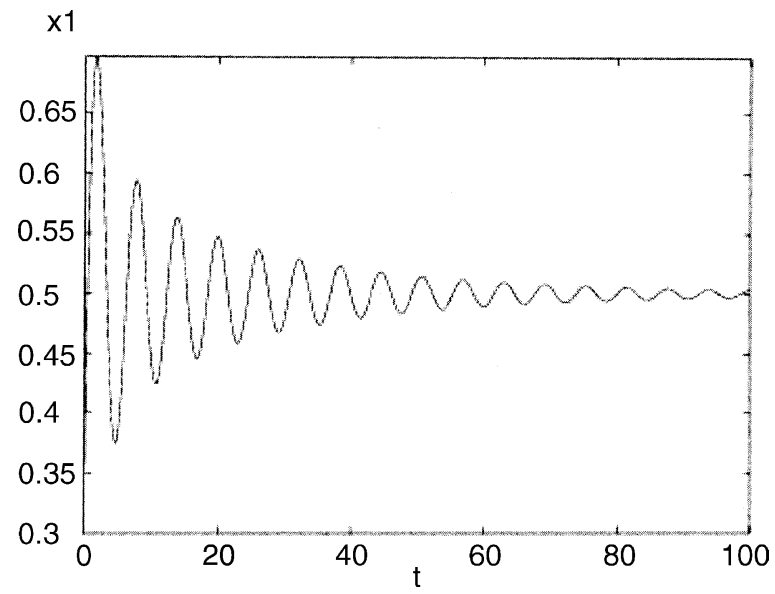

Fig. 1. Behavior of the first neuron in system (3.1) with small discrete delay: $a_{10}=1, a_{20}=2, b_{11}=-4, b_{22}=5, c_{12}=-5, c_{21}=4, f_{1}=4.5$, $f_{2}=-4.5$ and $\sigma_{s}=0.55$.

Then Eq. (5.9) becomes

$$
\lambda^{2}+A \lambda+D+E \mathrm{e}^{-\sigma \lambda}+F \lambda \mathrm{e}^{\sigma \lambda}=0 .
$$

Following the results in [22], we know that if (4.17) is satisfied and either

$$
D^{2}-E^{2}>0, \quad F^{2}-A^{2}+2 D<0
$$

or

$$
\left(F^{2}-A^{2}+2 D\right)^{2}<4\left(D^{2}-E^{2}\right),
$$

holds, then all roots of Eq. (5.12) have negative real parts for all discrete delays $\sigma_{12}+\sigma_{21}=\sigma \geq 0$. If

$$
D^{2}-E^{2}<0
$$

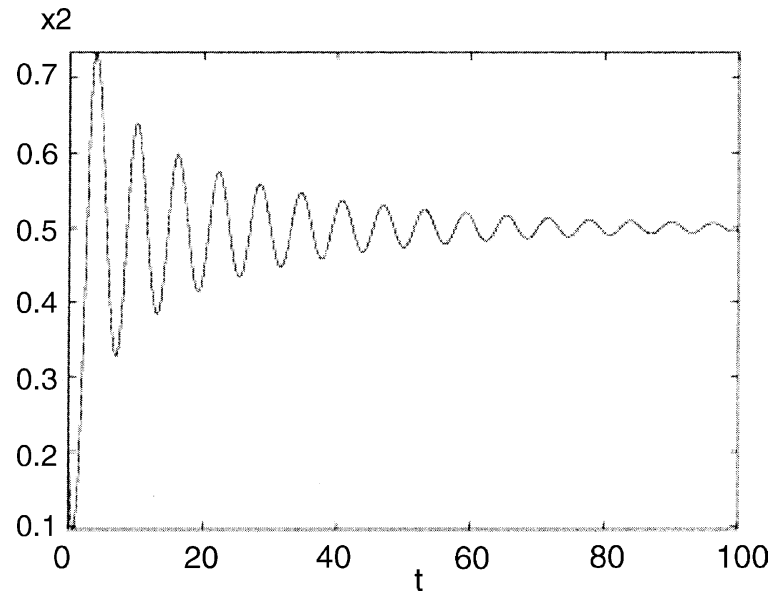

Fig. 2. Behavior of the second neuron in system (3.1) with small discrete delay: $a_{10}=1, a_{20}=2, b_{11}=-4, b_{22}=5, c_{12}=-5$, $c_{21}=4, f_{1}=4.5, f_{2}=-4.5$ and $\sigma_{s}=0.55$. 


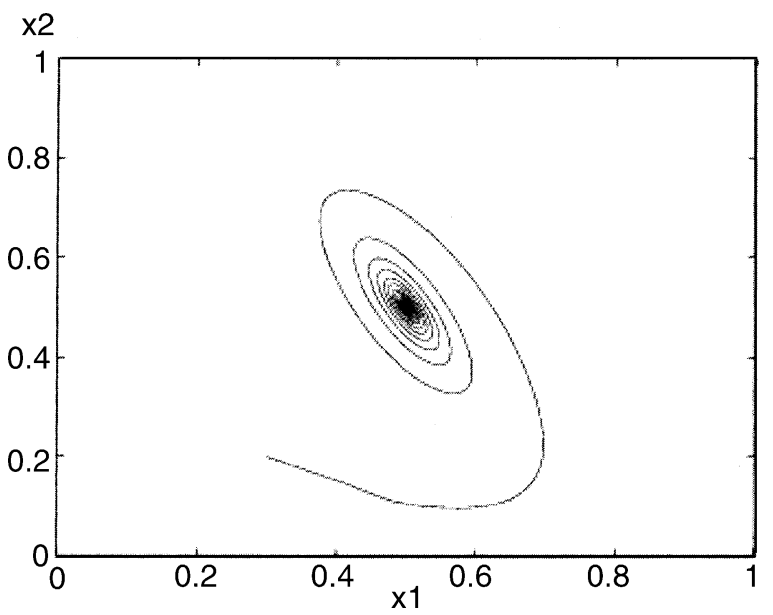

Fig. 3. Phase portrait of system (3.1) with small discrete delay: $a_{10}=1, a_{20}=2, b_{11}=-4, b_{22}=5, c_{12}=-5, c_{21}=4, f_{1}=4.5, f_{2}=-4.5$ and $\sigma_{s}=0.55$.

or

$$
F^{2}-A^{2}+2 D>0, \quad\left(F^{2}-A^{2}+2 D\right)^{2}=4\left(D^{2}-E^{2}\right)
$$

then Eq. (5.12) has a pair of purely imaginary roots $\pm \mathrm{i} \omega_{+}\left(\omega_{+}>0\right)$ when $\sigma=\sigma_{n, 1}^{1}$. If

$$
D^{2}-E^{2}>0, \quad F^{2}-A^{2}+2 D>0, \quad\left(F^{2}-A^{2}+2 D\right)^{2}>4\left(D^{2}-E^{2}\right)
$$

are satisfied, then Eq. (5.12) has one pair of purely imaginary roots $\pm \mathrm{i} \omega_{+}$, respectively $\pm \mathrm{i} \omega_{-}$, when $\sigma=\sigma_{n, 1}^{1}$, respectively $\sigma=\sigma_{n, 2}^{1}$, with $\omega_{+}>\omega_{-}>0$, where

$$
\omega_{ \pm}^{2}=\frac{1}{2}\left(F^{2}-A^{2}+2 D\right) \pm\left[\frac{1}{4}\left(F^{2}-A^{2}+2 D\right)^{2}-\left(D^{2}-E^{2}\right)\right]^{1 / 2},
$$

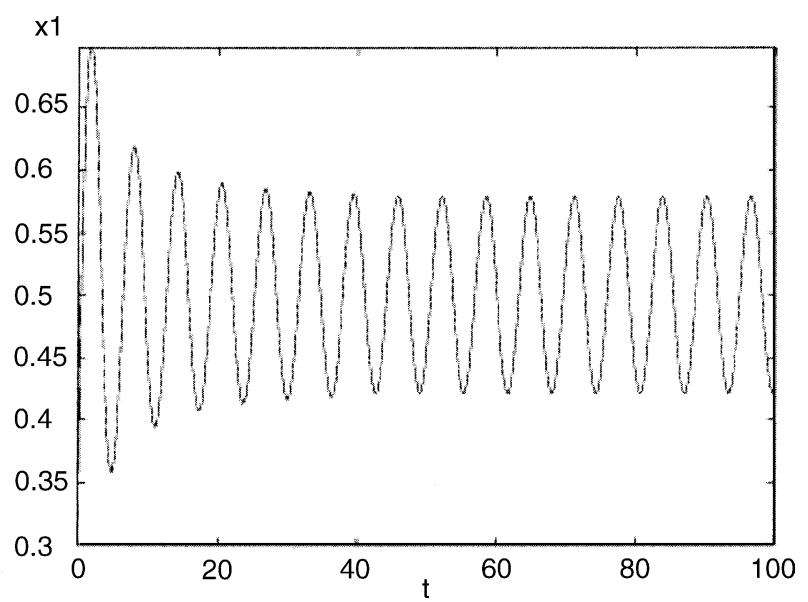

Fig. 4. Behavior of the first neuron in system (3.1) with large discrete delay: $a_{10}=1, a_{20}=2, b_{11}=-4, b_{22}=5, c_{12}=-5, c_{21}=4, f_{1}=4.5$, $f_{2}=-4.5$ and $\sigma_{s}=0.65$. 


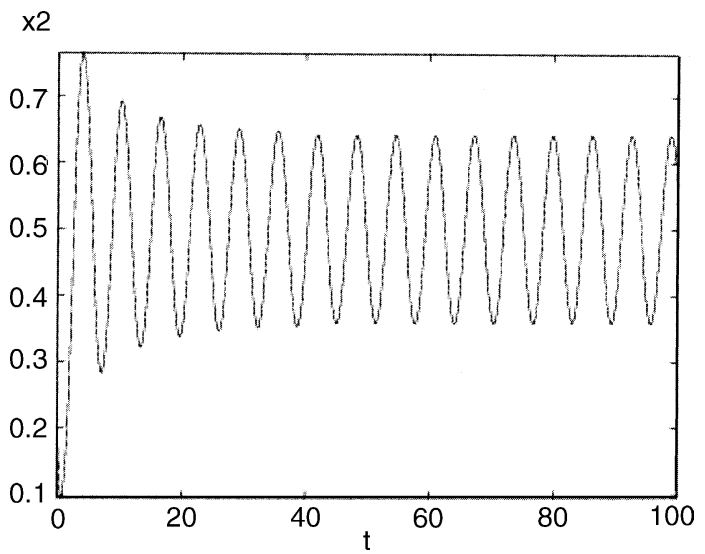

Fig. 5. Behavior of the second neuron in system (3.1) with large discrete delay: $a_{10}=1, a_{20}=2, b_{11}=-4, b_{22}=5, c_{12}=-5, c_{21}=4$, $f_{1}=4.5, f_{2}=-4.5$ and $\sigma_{s}=0.65$.

$$
\begin{aligned}
& \sigma_{n, 1}^{1}=\frac{1}{\omega_{+}} \cos ^{-1}\left\{\frac{E\left(\omega_{+}^{2}-D\right)-A F \omega_{+}^{2}}{F^{2} \omega_{+}^{2}+E^{2}}\right\}+\frac{2 n \pi}{\omega_{+}}, \\
& \sigma_{n, 2}^{1}=\frac{1}{\omega_{-}} \cos ^{-1}\left\{\frac{E\left(\omega_{-}^{2}-D\right)-A F \omega_{-}^{2}}{F^{2} \omega_{-}^{2}+E^{2}}\right\}+\frac{2 n \pi}{\omega_{-}} \quad(n=0,1,2, \ldots) .
\end{aligned}
$$

Therefore, when there is only one pair of purely imaginary root $\pm \mathrm{i} \omega_{+}$, only crossing of the imaginary axis from left to right is possible as $\sigma$ increases, and stability of the zero solutions can only be lost but not regained. When there are two pairs of purely imaginary roots $\pm \mathrm{i} \omega_{ \pm}$, crossing of the imaginary axis from left to right occurs whenever $\sigma$ assumes a value corresponding to $\omega_{+}$and crossing of the imaginary axis from right to left occurs whenever $\sigma$ assumes a value corresponding to $\omega_{-}$.

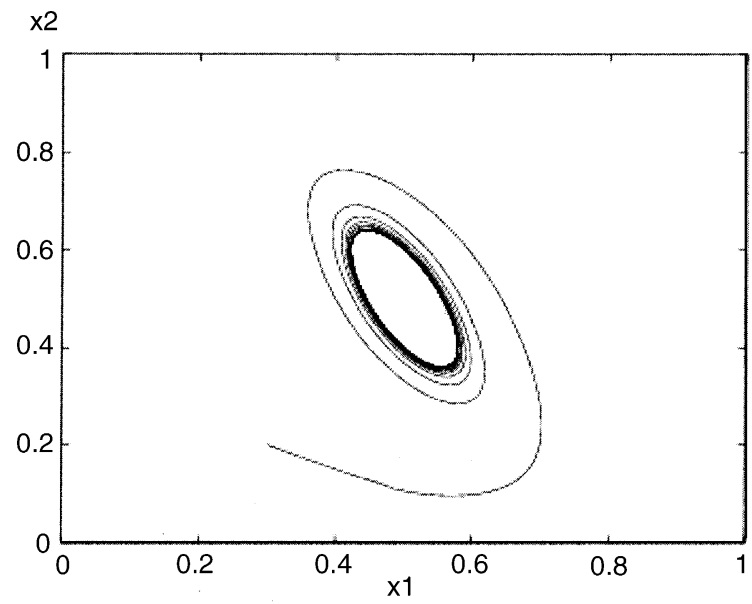

Fig. 6. Phase portrait of system (3.1) with large discrete delay: $a_{10}=1, a_{20}=2, b_{11}=-4, b_{22}=5, c_{12}=-5, c_{21}=4, f_{1}=4.5, f_{2}=-4.5$ and $\sigma=0.65$. 


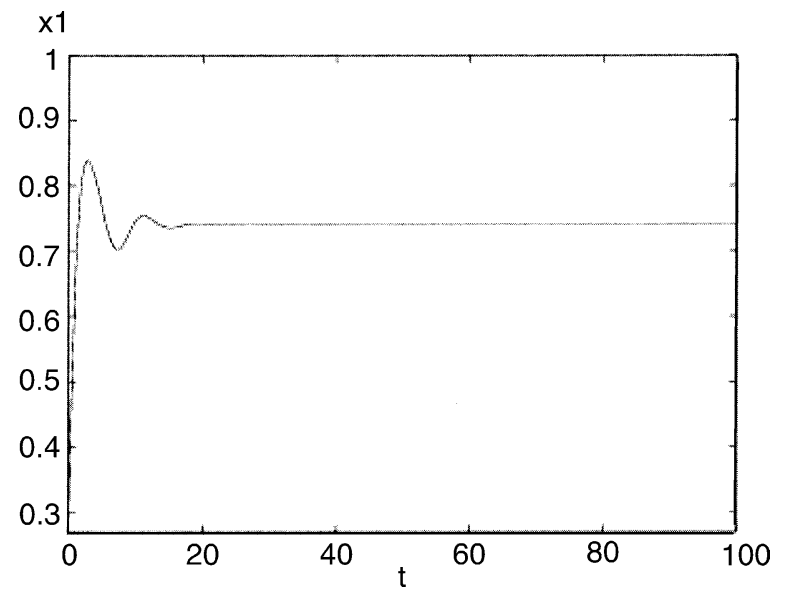

Fig. 7. Behavior of the first neuron in system (4.1) with large distributed delay coefficient: $a_{10}=a_{20}=1, b_{11}=-10, b_{22}=-7$, $c_{12}=8, c_{21}=12, f_{1}=1, f_{2}=-2.5$ and $\alpha_{11}=\alpha_{22}=1.5$.

Note that if the system (5.4) is stable for $\sigma=0$, then necessarily $\sigma_{0,1}^{1}<\sigma_{0,2}^{1}$ (since the multiplicity of roots with positive real parts cannot become negative). Since

$$
\sigma_{n+1,1}^{1}-\sigma_{n, 1}^{1}=\frac{2 \pi}{\omega_{+}}<\frac{2 \pi}{\omega_{-}}=\sigma_{n+1,2}^{1}-\sigma_{n, 2}^{1},
$$

there can exist only a finite number of switches between stability and instability. Hence, there exist $k$ switches from stability to instability to stability when the parameters are such that

$$
\sigma_{0,1}^{1}<\sigma_{0,2}^{1}<\sigma_{1,1}^{1}<\sigma_{1,2}^{1}<\cdots<\sigma_{k-1,1}^{1}<\sigma_{k-1,2}^{1}<\sigma_{k, 1}^{1}<\sigma_{k+1,1}^{1}<\sigma_{k, 2}^{1}<\cdots,
$$

where $k$ is an integer.

Now we shall consider the negative case in Eq. (5.8)

$$
\left[\left(a_{10}+\lambda\right)\left(a_{1}+\lambda\right)-B_{1}\right]-\sqrt{C}\left(a_{1}+\lambda\right) \mathrm{e}^{-(1 / 2)\left(\sigma_{12}+\sigma_{21}\right) \lambda}=0 .
$$

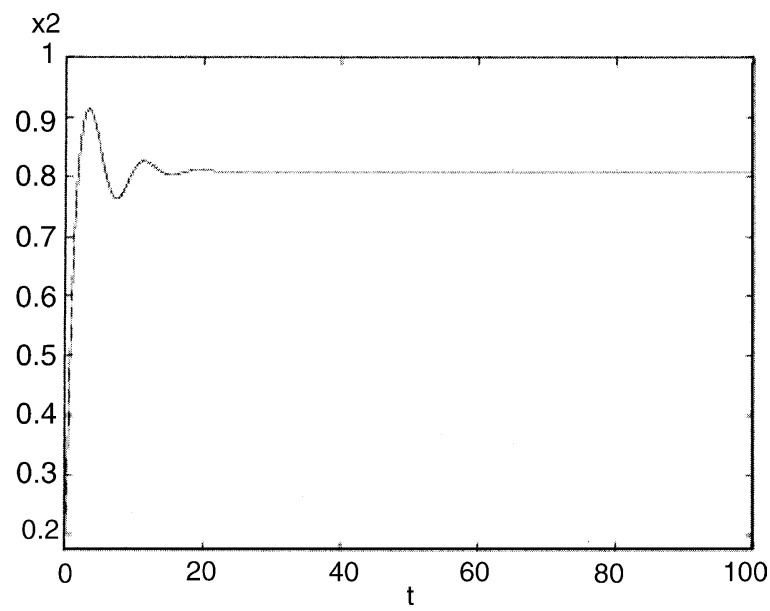

Fig. 8. Behavior of the second neuron in system (4.1) with large distributed delay coefficient: $a_{10}=a_{20}=1, b_{11}=-10, b_{22}=-7$, $c_{12}=8, c_{21}=12, f_{1}=1, f_{2}=-2.5$ and $\alpha_{11}=\alpha_{22}=1.5$. 


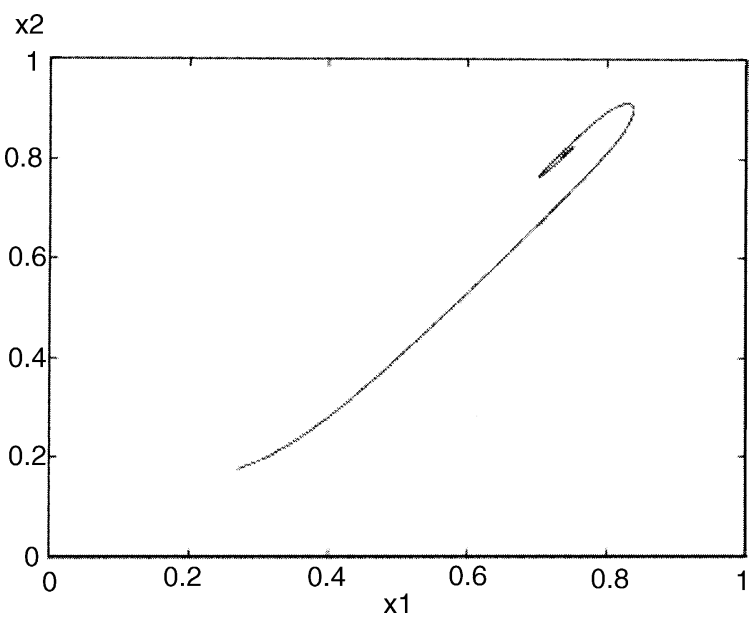

Fig. 9. Phase portrait of system (4.1) with large distributed delay coefficient: $a_{10}=a_{20}=1, b_{11}=-10, b_{22}=-7, c_{12}=8, c_{21}=12, f_{1}=1$, $f_{2}=-2.5$ and $\alpha_{11}=\alpha_{22}=1.5$.

Using the same notation used for the positive case, Eq. (5.22) becomes

$$
\lambda^{2}+A \lambda+D-E \mathrm{e}^{-\sigma \lambda}-F \lambda \mathrm{e}^{-\sigma \lambda}=0 .
$$

Similar to the positive case, when (4.14) and (4.15) are satisfied and either (5.13) or (5.14) holds, then all roots of Eq. (5.23) have negative real parts for all discrete delays $\sigma_{12}+\sigma_{21}=\sigma \geq 0$. If (5.15) or (5.16) holds, then Eq. (5.23) has one pair of purely imaginary roots $\pm \mathrm{i} v_{+}\left(v_{+}>0\right)$ when $\sigma=\sigma_{m, 1}^{2}$. If (5.17) holds, then Eq. (5.23) has one pair of purely imaginary roots $\pm \mathrm{i} v_{+}$, respectively $\pm \mathrm{i} v_{-}$, when $\sigma=\sigma_{m, 1}^{2}$, respectively $\sigma=\sigma_{m, 2}^{2}$, with $v_{+}>v_{-}>0$, where

$$
v_{ \pm}^{2}=\frac{1}{2}\left(F^{2}-A^{2}+2 D\right) \pm\left[\frac{1}{4}\left(F^{2}-A^{2}+2 D\right)^{2}-\left(D^{2}-E^{2}\right)\right]^{1 / 2},
$$

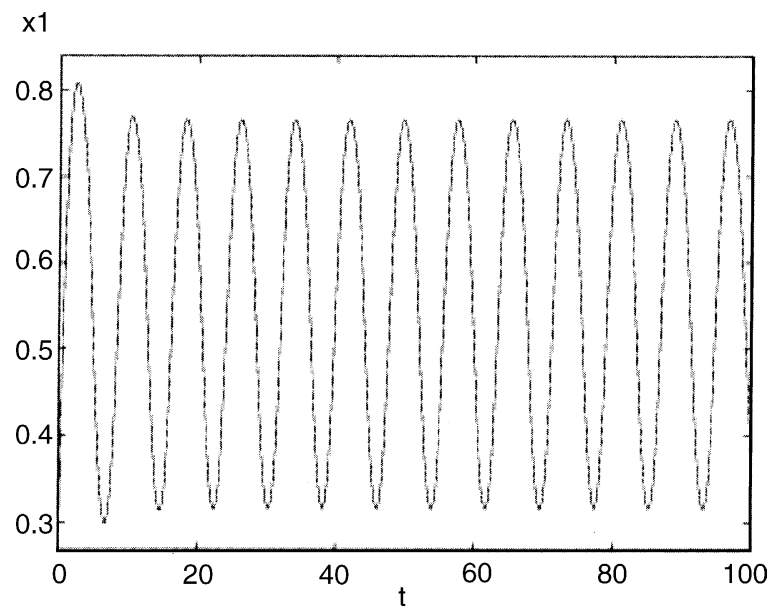

Fig. 10. Behavior of the first neuron in system (4.1) with small distributed delay coefficient: $a_{10}=a_{20}=1, b_{11}=-10, b_{22}=-7, c_{12}=8$, $c_{21}=12, f_{1}=1, f_{2}=-2.5$ and $\alpha_{11}=\alpha_{22}=1.3$. 


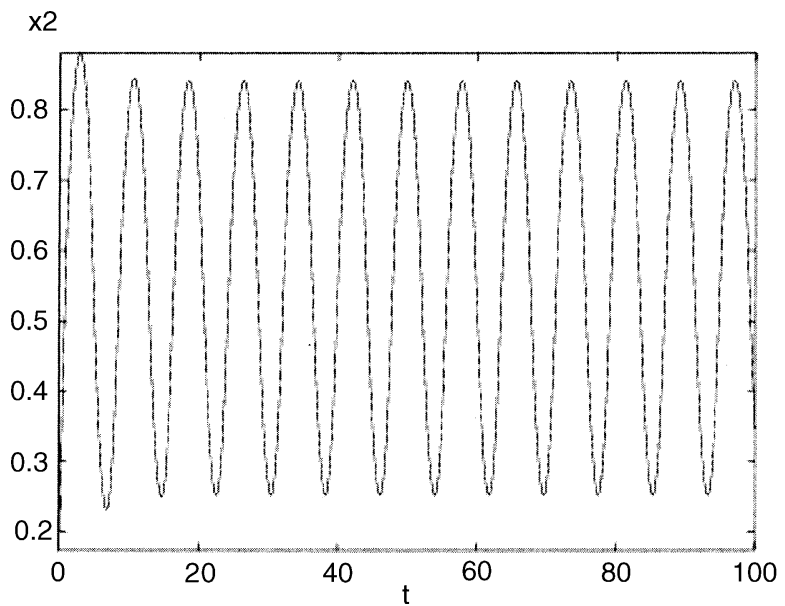

Fig. 11. Behavior of the second neuron in system (4.1) with small distributed delay coefficient: $a_{10}=a_{20}=1, b_{11}=-10, b_{22}=-7$, $c_{12}=8, c_{21}=12, f_{1}=1, f_{2}=-2.5$ and $\alpha_{11}=\alpha_{22}=1.3$.

$$
\begin{aligned}
& \sigma_{n, 1}^{2}=\frac{1}{v_{+}} \cos ^{-1}\left\{\frac{A F v_{+}^{2}-E\left(v_{+}^{2}-D\right)}{F^{2} v_{+}^{2}+E^{2}}\right\}+\frac{2 n \pi}{v_{+}} \\
& \sigma_{n, 2}^{2}=\frac{1}{v_{-}} \cos ^{-1}\left\{\frac{A F v_{-}^{2}-E\left(v_{-}^{2}-D\right)}{F^{2} v_{-}^{2}+E^{2}}\right\}+\frac{2 n \pi}{v_{-}} \quad(n=0,1,2, \ldots) .
\end{aligned}
$$

with $(k$ is an integer)

$$
\sigma_{0,1}^{2}<\sigma_{0,2}^{2}<\sigma_{1,1}^{2}<\sigma_{1,2}^{2}<\cdots<\sigma_{k-1,1}^{2}<\sigma_{k-1,2}^{2}<\sigma_{k, 1}^{2}<\sigma_{k+1,1}^{2}<\sigma_{k, 2}^{2}<\cdots .
$$

The stability and bifurcation results of (5.1) can be summarized in the following theorem.

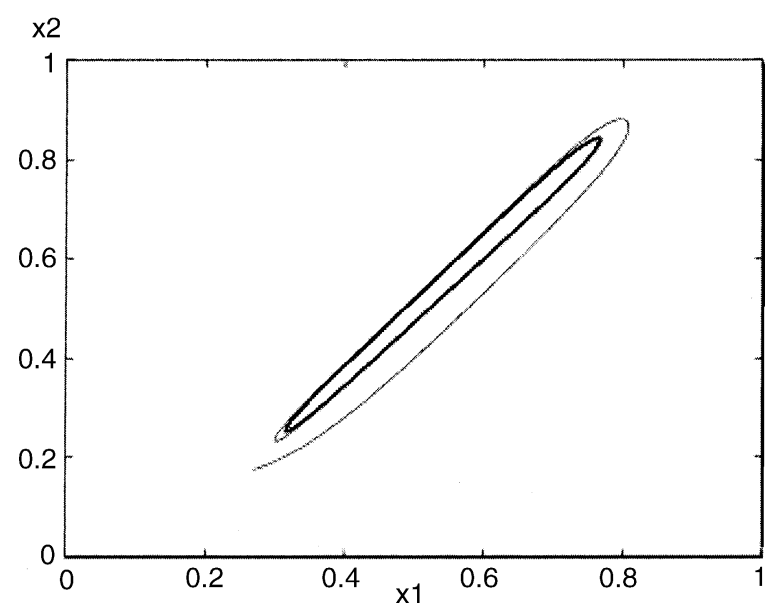

Fig. 12. Phase portrait of system (4.1) with small distributed delay coefficient: $a_{10}=a_{20}=1, b_{11}=-10, b_{22}=-7, c_{12}=8, c_{21}=12$, $f_{1}=1, f_{2}=-2.5$ and $\alpha_{11}=\alpha_{22}=1.3$. 


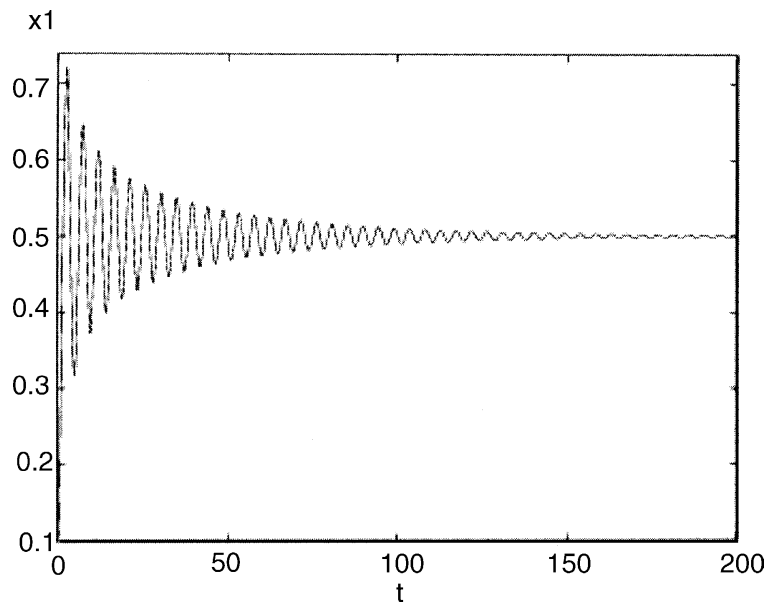

Fig. 13. Behavior of the first neuron in system (5.1) with small discrete delay: $a_{10}=a_{20}=1, b_{11}=b_{22}=-4, c_{12}=c_{21}=7, \alpha_{11}=\alpha_{22}=1$, $f_{1}=f_{2}=-1.5$ and $\sigma=0.6$.

Theorem 5.1. Assume the two neurons are identical in system (5.1). Suppose conditions (4.14) (4.15) and (4.17) are satisfied.

1. If (5.13) and (5.14) hold, then the steady state $\left(x_{1}^{*}, x_{2}^{*}\right)$ of (5.1) is asymptotically stable for all discrete delays $\sigma_{12}+\sigma_{21}=\sigma \geq 0$.

2. If (5.15) or (5.16) holds, $\sigma \in\left[0, \min \left\{\sigma_{0,1}^{1}, \sigma_{0,1}^{2}\right\}\right)$, then the steady-state $\left(x_{1}^{*}, x_{2}^{*}\right)$ of $(5.1)$ is asymptotically stable. If $\sigma>\min \left\{\sigma_{0,1}^{1}, \sigma_{0,1}^{2}\right\}$, then the steady-state $\left(x_{1}^{*}, x_{2}^{*}\right)$ of $(5.1)$ is unstable. $\sigma_{n, 1}^{1}$ and $\sigma_{n, 1}^{2}(n=0,1,2, \ldots)$ are Hopf bifurcation values of system (5.1).

3. If (5.17) holds, $\sigma \in\left[0, \min \left\{\sigma_{1,0}^{1}, \sigma_{1,0}^{2}\right\}\right)$, then the steady-state $\left(x_{1}^{*}, x_{2}^{*}\right)$ of $(5.1)$ is asymptotically stable. There is a positive integer $k$ such that there are $k$ switches from stability to instability and back to stability, that is, when $\sigma \in\left[\min \left\{\sigma_{n, 1}^{1}, \sigma_{n, 1}^{2}\right\}, \max \left\{\sigma_{n, 2}^{1}, \sigma_{n, 2}^{2}\right\}\right)$ the steady-state $\left(x_{1}^{*}, x_{2}^{*}\right)$ of $(5.1)$ is unstable; when $\sigma \in\left[\max \left\{\sigma_{n, 2}^{1}, \sigma_{n, 2}^{2}\right\}\right.$,

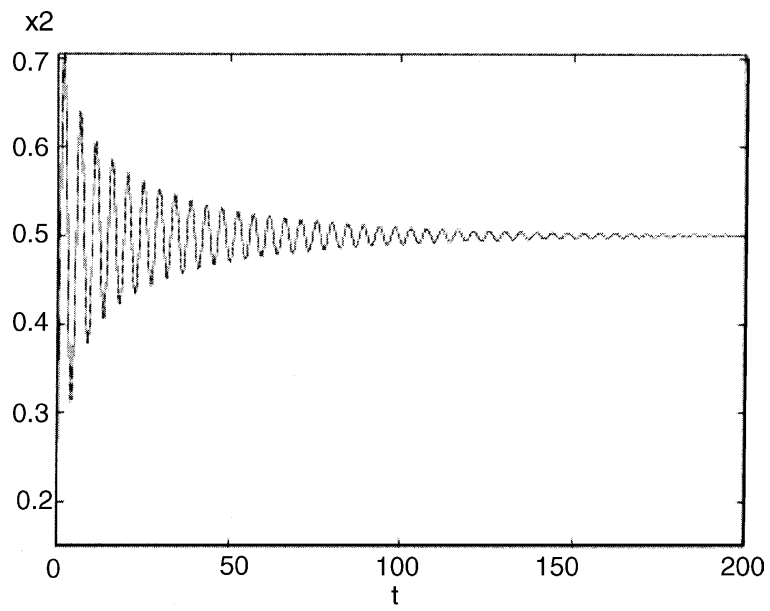

Fig. 14. Behavior of the second neuron in system (5.1) with small discrete delay: $a_{10}=a_{20}=1, b_{11}=b_{22}=-4, c_{12}=c_{21}=7$, $\alpha_{11}=\alpha_{22}=1, f_{1}=f_{2}=-1.5$ and $\sigma=0.6$. 
$\left.\min \left\{\sigma_{n+1,1}^{1}, \sigma_{n+1,1}^{2}\right\}\right)$ the steady-state $\left(x_{1}^{*}, x_{2}^{*}\right)$ of $(5.1)$ is asymptotically stable. If $\sigma>\min \left\{\sigma_{k, 1}^{1}, \sigma_{k, 1}^{2}\right\}$, then the steady-state $\left(x_{1}^{*}, x_{2}^{*}\right)$ of $(5.1)$ is unstable.

\section{Numerical examples}

In this section, we illustrate the validity of the results by considering examples of artificial neural networks corresponding to each of the special. All numerical simulations are carried out using the computer program XPP [6].

Consider an example of a two-neuron network with neural interaction history and instantaneous feedback, system (3.1), with $a_{10}=1, a_{20}=2, b_{11}=-4, b_{22}=5, c_{12}=-5, c_{21}=4, f_{1}=4.5$, and $f_{2}=-4.5$. According to the

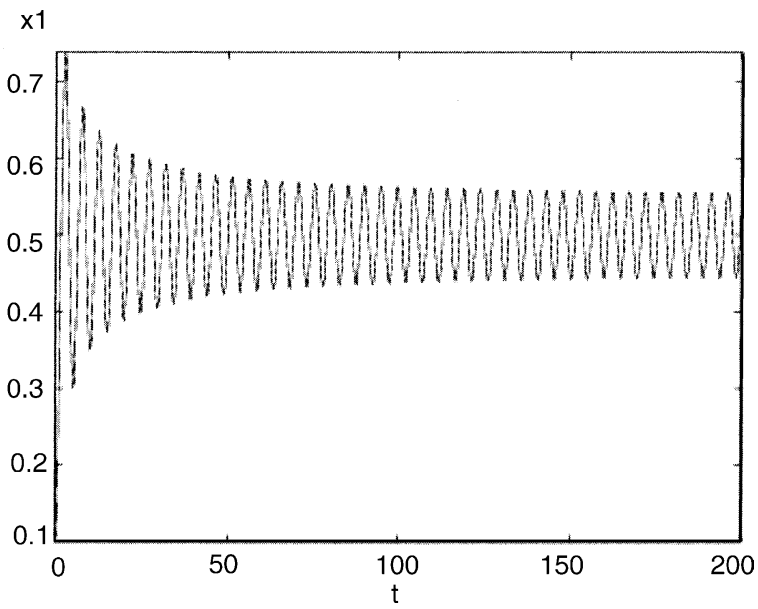

Fig. 15. Behavior of the first neuron in system (5.1) with large discrete delay: $a_{10}=a_{20}=1, b_{11}=b_{22}=-4, c_{12}=c_{21}=7, \alpha_{11}=\alpha_{22}=1$, $f_{1}=f_{2}=-1.5$ and $\sigma=1$.

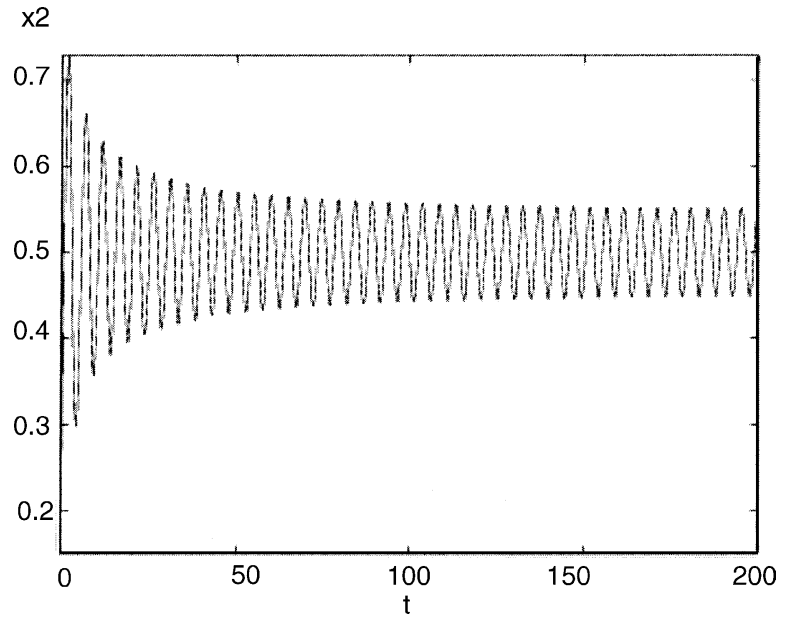

Fig. 16. Behavior of the second neuron in system (5.1) with large discrete delay: $a_{10}=a_{20}=1, b_{11}=b_{22}=-4, c_{12}=c_{21}=7$, $\alpha_{11}=\alpha_{22}=1, f_{1}=f_{2}=-1.5$ and $\sigma=1$. 


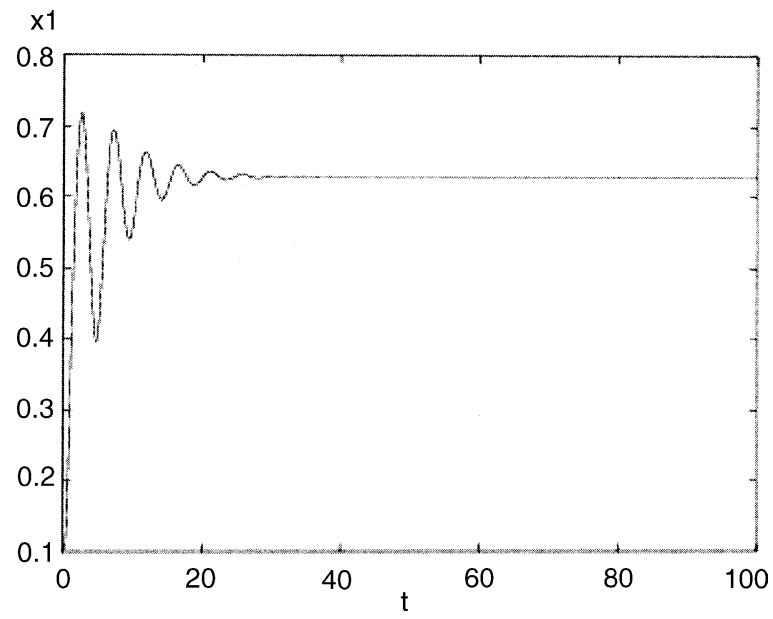

Fig. 17. Behavior of the first neuron in system (5.1) with small discrete delay and small distributed delay: coefficient $a_{10}=a_{20}=1$, $b_{11}=b_{22}=-4, c_{12}=c_{21}=7, \alpha_{11}=\alpha_{22}=0.8, f_{1}=f_{2}=-1.5$ and $\sigma=0.6$.

parameters chosen, we find that the steady-state solution is $(0.5,0.5)$ and the critical discrete delay is $\sigma_{s, 0}=0.6$. Choose the delays $\sigma_{12}=0.35$ and $\sigma_{21}=0.20$, then $\sigma_{12}+\sigma_{21}<0.6$. Figs. 1-3 show that the steady-state solution is asymptotically stable. By Theorem 3.1, a Hopf bifurcation occurs when $\sigma_{12}+\sigma_{21}=0.6$, and the steady-state solution loses its stability for $\sigma_{12}+\sigma_{21}>0.6$. By choosing $\sigma_{12}=0.35$ and $\sigma_{21}=0.30$, it is observed that the computer simulations in Figs. 4-6 support the results from Theorem 3.1.

In a two-neuron network with delayed neural feedback and no neural interaction history, the stability of the system around its steady-state solution is governed by the distributed delay coefficient parameter. Consider system (4.1) with $a_{10}=a_{20}=1, b_{11}=-10, b_{22}=-7, c_{12}=8, c_{21}=12, f_{1}=1$ and $f_{2}=-2.5$. By using (4.3) and $\Psi\left(\alpha_{11}\right)=0$ respectively, we find the steady-state solution to be $(0.5,0.5)$ and the critical value for the distributed delay coefficient to be $\alpha_{11}^{*}=1.4$. Figs. 7-9 show the asymptotic stability of the steady-state solution when the distributed delay coefficients are chosen such that $\alpha_{11}=\alpha_{22}=1.5>\alpha_{11}^{*}$. When the distributed delay coefficient

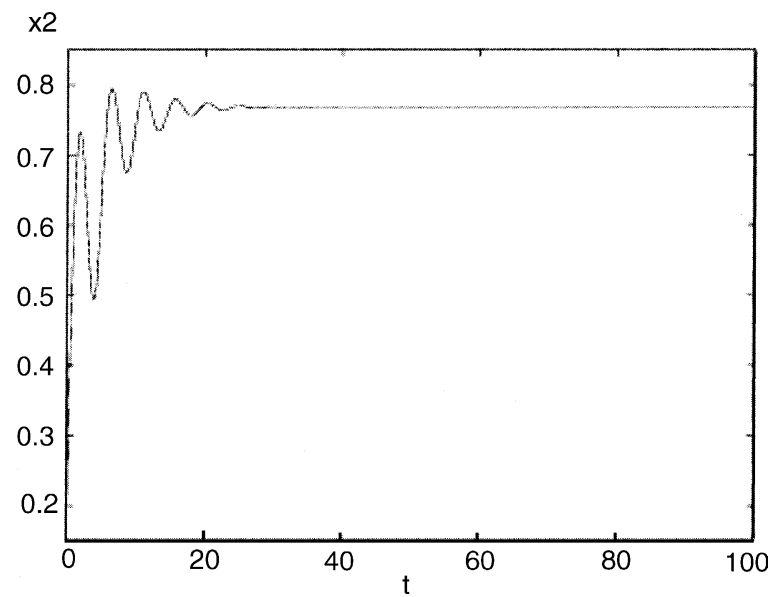

Fig. 18. Behavior of the second neuron in system (5.1) with small discrete delay and small distributed delay: coefficient $a_{10}=a_{20}=1$, $b_{11}=b_{22}=-4, c_{12}=c_{21}=7, \alpha_{11}=\alpha_{22}=0.8, f_{1}=f_{2}=-1.5$ and $\sigma=0.6$. 
decreases and passes through the critical value $\alpha_{11}^{*}$, Hopf bifurcation occurs; i.e. a family of periodic solutions bifurcates from the steady-state solution. This is illustrated by Figs. $10-12$ where $\alpha_{11}=\alpha_{22}=1.3$.

Finally, we consider an artificial neural network consisting of two neurons with delayed neural feedback and neural interaction history. Consider system (5.1) with $a_{10}=a_{20}=1, b_{11}=b_{22}=-4, c_{12}=c_{21}=7, \alpha_{11}=\alpha_{22}=1$, and $f_{1}=f_{2}=-1.5$. The steady-state solution is $(0.5,0.5)$ and the critical discrete delay is $\sigma_{0,1}^{1}=0.8$. Choosing $\sigma_{12}=0.5$ and $\sigma_{21}=0.7$, then we have $\sigma=\left(\sigma_{12}+\sigma_{21}\right) / 2<0.8$. Figs. 13 and 14 show the asymptotic stability of the steady-state solution. By Theorem 5.1, Hopf bifurcation occurs when $\left(\sigma_{12}+\sigma_{21}\right) / 2=\sigma_{0,1}^{1}$ and the stability of the steady-state solution is lost once the total delay passes this critical value. This is illustrated in Figs. 15 and 16 where $\sigma=1$. When the total discrete delay in system (5.1) is less than the critical value and the distributed delay coefficient is altered to give large distributed delay, it is seen that the stability of the system is unchanged. This means that the behavior of system (5.1) is governed by the discrete delay. Figs. 17 and 18 show the asymptotic stability of the steady-state solution when the parameters are chosen such that $a_{10}=a_{20}=1, b_{11}=b_{22}=-4, c_{12}=c_{21}=$ $7, \alpha_{11}=\alpha_{22}=0.8, f_{1}=f_{2}=-1.5$ and $\sigma=0.6$.

\section{Conclusions}

Recently, a variety of artificial models have been established to describe neural networks with discrete delays, distributed delays, or with both sharp and continuous delays [1-3,7-16,21,23,25-28]. A few sufficient criteria have been established for stability of neural models with discrete or distributed delays [8-11,26,28]. Bifurcation analysis has also been carried out for neural network models with multiple discrete delays or distributed delays $[1-3,7,9,11,15,21,23,27,28]$. However, there are few papers on the stability and bifurcation of neural network models with both discrete and distributed delays.

In this paper, we have considered a two-neuron network model with multiple discrete and distributed delays. The distributed delays arose from the neural feedback while the discrete delays arose from the neural interaction history. Stability of the steady-state solutions and the oscillation around the steady-state solutions have been studied. The existence of oscillations around the steady-state solutions has been shown via Hopf bifurcation analysis.

Three special cases of the generalized artificial neural network have been considered in Sections 3-5. In the case of neural interactions with instantaneous feedback and neural interaction history, we found that when the sum of the two delays, $\sigma_{12}+\sigma_{21}=\sigma_{s}$, varies, the steady-state solution loses its stability and Hopf bifurcation occurs, that is, a family of periodic solutions bifurcates from the steady-state solution when $\sigma_{s}$ passes a critical value. In the case of neural interactions with delayed neural feedback and no neural interaction history, we obtained conditions for stability of the artificial neural network. Furthermore, using the average delay as the bifurcation parameter, we gave the conditions under which Hopf bifurcation would occur in the neural network model. In the case of neural interactions with delayed neural feedback and neural interaction history, it was found through numerical simulations that the distributed delay in the system is dominated by the discrete delay. We established conditions on the average discrete delay in the system, $\sigma$, under which the stability of the neural system is maintained. Stability switches and bifurcation were studied by applying the results in $[4,22]$.

In all of the cases considered, it is seen that once the critical delay is reached the two neurons in the system enter an oscillating behavior in which the firing rate of each neuron adapts the other neuron by inhibiting or exciting its firing rate. In the case of neural interaction with delayed neural feedback and neural interaction history, it was found that the stability results of a system with two inhibitory connections are identical to those of a system with two excitatory connections of the same magnitude.

Neural networks with delays exhibit very rich dynamics. From the point of view of nonlinear dynamics, analyzing these neural networks is useful in solving problems of both theoretical and practical importance. Due to the 
complexity of large neural models, stability analysis is carried out for small neural networks. Such analysis is potentially useful since the complexity found in these small network models might be carried over to larger networks. They may also serve as first approximations in modeling some simple invertebrate patterns. Also, by considering the minimum requirements for a particular pattern of activity, we can gain some insight into why some nervous systems have so great a number of neural elements.

\section{Acknowledgements}

Research was partially supported by the Natural Science and Engineering Research Council (NSERC) of Canada and a start-up grant from the College of Arts and Sciences at the University of Miami. We are grateful to the two anonymous referees for their careful reading and constructive comments and suggestions which helped improving the presentation of the paper.

\section{References}

[1] K.L. Babcock, R.M. Westervelt, Dynamics of simple electronic neural networks, Physica D 28 (1987) 305-316.

[2] T.A. Burton, Volterra Integral and Differential Equations, Academic Press, New York, 1983.

[3] Y. Chen, J. Wu, Minimal instability and unstable set of a phase-locked periodic orbit in a delayed neural network, Physica D 134 (1999) $185-199$.

[4] K.L. Cooke, Z. Grossman, Discrete delays, distributed delay and stability switches, J. Math. Anal. Appl. 86 (1982) $592-627$.

[5] J.D. Cowan, A statistical mechanics of neuron activity, in: M. Gerstenhaber (Ed.), Some Mathematical Questions in Biology, American Mathematical Society, Providence, RI, 1970, pp. 1-58.

[6] B. Ermentrout, XPPAUT5.0—-The Differential Equations Tool, University of Pittsburgh, Pittsburgh, 2001.

[7] T. Faria, On a planar system modeling a neuron network with memory, J. Diff. Eqns. 168 (2000) 129-149.

[8] S. Gao, L. Huang, Exponential stability and periodic solutions of neural networks with continuously distributed delays, Phys. Rev. E 67 (2003) 011902; erratum 069901.

[9] F. Giannakopoulos, A. Zapp, Bifurcations in a planar system of differential delay equations modeling neural activity, Physica D 159 (2001) $215-232$.

[10] K. Gopalsamy, X.-Z. He, Stability in asymmetric Hopfield nets with transmission delays, Physica D 76 (1994) $344-358$.

[11] K. Gopalsamy, I. Leung, Delay-induced periodicity in a neural netlet of excitation and inhibition, Physica D 89 (1996) $395-426$.

[12] J.K. Hale, S.M. Verduyn Lunel, Introduction to functional differential equations, in: Applied Mathematics Sciences, vol. 99, Springer-Verlag, New York, 1993.

[13] J.J. Hopfield, Neurons with graded response have collective computational properties like those of two-state neurons, Proc. Natl. Acad. Sci. U.S.A. 81 (1984) 3088-3092.

[14] D. Koruga, Molecular networks as a sub-neural factor of neural networks, BioSystems 23 (1990) 297-303.

[15] X. Liao, K.-W. Wong, Z. Wu, Bifurcation analysis on a two-neuron system with distributed delays, Physica D 149 (2001) $123-141$.

[16] X. Liao, Z. Wu, J. Yu, Stability switches and bifurcation analysis of neural network with continuously delay, IEEE Trans. Syst. Man. Cybernet. 29 (1999) 692-696.

[17] N. MacDonald, Time Lag in Biological Models, Lecture Notes in Biomathematics 27, Springer-Verlag, Heidelberg, 1978.

[18] R.K. Miller, Nonlinear Volterra Integral Equations, Benjamin, New York, 1971.

[19] M.N. Ogûztöreli, Activity analysis of neural networks, Biol. Cybernet. 34 (1979) 159-169.

[20] M.N. Ogûztöreli, G.M. Steil, T.M. Caelli, Control mechanisms of a neural network, Biol. Cybernet. 54 (1986) 21-28.

[21] L. Olien, J. Belair, Bifurcation, stability and monotonicity properties of a delayed neural network model, Physica D 102 (1997) 349-363.

[22] S. Ruan, Absolute stability, conditional stability and bifurcation in Kolmogorov-type predator-prey systems with discrete delays, Quart. Appl. Math. 59 (2001) 159-173.

[23] S. Ruan, J. Wei, Periodic solutions of planar systems with two delays, Proc. Roy. Soc. Edinburgh, Section A 129 (1999) $1017-1032$.

[24] P. Sokolove, Computer simulation of after-inhibition in crayfish slowly adapting stretch receptor, Biophys. J. 12 (1972) $1429-1451$.

[25] D.W. Tank, J.J. Hopfield, Neural computation by concentrating information in time, Proc. Natl. Acad. Sci. U.S.A. 84 (1987) $1896-1991$.

[26] L. Wang, X. Zou, Harmless delays in Cohen-Grossberg neural networks, Physica D 170 (2002) 162-173.

[27] J. Wei, S. Ruan, Stability and bifurcation in a neural network model with two delays, Physica D 130 (1999) $255-272$.

[28] J. Wu, Introduction to Neural Dynamics and Signal Transmission Delay, Walter de Gruyter, Berlin, 2001. 